steeply? It was probably a cultural change associated with changing public attitudes towards orthodox medicine. But in spite of puzzling about it for years I can produce no convincing answers.

\section{Irvine Loudon}

\section{REFERENCES}

1. Archives of the Radcliffe Infirmary, Oxford.

2. Rivington W. The medical profession. Dublin: Fannin and Co., 1879.

3. Burdett H. Hospitals and asylums of the world. London: Churchill, 1893.

4. Archives of Westminister Hospital, London. Annual reports.

5. Bridges R. An account of the Casualty Department. St.
Bartholomew's Hospital Reports, 1878; 14: 167-182.

7. King's Hospital Fund for London. Report of the Committee appointed to inquire into the system prevailing in the London Hospitals with regard to admission to out-patients. London: King's Hospital Fund for London, 1912.

DOI: 10.3399/bjgp08X277113

\title{
COMMENTARY
}

The theological justification of general practice usually stresses personal continuing care and tri-partite diagnosis. In reality the main scientific basis for having generalists practising in the community is because gatekeeping is an effective way to increase the prevalence of serious disease in the population of patients whom hospital doctors see. Since a high prevalence of disease in a population ensures that the positive predictive value of signs and symptoms is increased, having effective gatekeepers turns out to make the diagnostic task of hospital doctors easier. And by the same token enables GPs to be much better at diagnosing normalcy - 'I don't know what's wrong with you but it isn't serious' is predicated on the fact that an absent sign or symptom has a high negative predictive value in low prevalence populations. Gatekeeping might have started out as a restrictive practice but hey! it was actually us being really cool on behalf of our patients. So everyone's happy and health systems where generalists restrict referrals turn out to be more efficient than those without. ${ }^{1}$

But what about gatekeeping today? Now that we finally understand just how important gatekeeping is for efficient care is that the end of the story? Turns out that we can, and should, be doing much better. Suppose that in your next surgery you see a 50-year-old white woman of BMI 30 with three episodes of vaginal bleeding over the last 15 months. Deciding whether she needs referral would be much easier if we knew the predictive value of this given set of signs and symptoms in this particular population of women when seen in the community.

Such finely-tuned predictive values are within our grasp - indeed, they have been so for several years had we bestirred ourselves. All we need is a significant number of practices to log $100 \%$ of all relevant symptoms and then record $100 \%$ of all relevant diagnoses and outcomes. Plus a great deal of commitment and hard work. At scale and with up to 1 million consultations per day clicking through the counters, this would progressively turn our collective diagnostic skills, intuitions, and failings into a set of evermore refined and wideranging positive and negative predictive values for community-based symptom complexes. Such a system could ultimately provide the consulting clinician with evidence-based statements of probable disease risk that incorporated genetic, demographic, and symptom variables tuned to each individual in front of them. In time it could also incorporate the prior probabilities for diagnostic tests of interest and link particular treatments with actual outcomes.

Would this mean the end of gatekeeping? That depends on whether it would still be useful to have diagnosis carried out in those highprevalence settings that we currently call hospitals. In short, whether it is economically worthwhile to have something on the other side of the gate.

Ironically, and by the back door, we have come full circle to some kind of 'personalised' care. But now 'personal' does not mean what the patient as a person desires but what particular combination of tests and treatments is likely to most benefit this individual collection of demographic, genetic, and symptom complex risks that is currently consulting with me. ${ }^{2}$

Such a vision is an example of how the new informational fabrics that are enveloping our world can be used to link very large scale social networks with massed data into entirely new solutions. In this new world Google constitutes our swaddling clothes, wrapping us in any information we desire. GPS is our baby bouncer, guiding us through an always-mapped universe. The NHS with its 1 million GP consultations a day clocking through the new electronic record (I'm an optimist) constitutes the substrate out of which we could, if we wished, create a medical informational fabric of equal import to Google and GPS. One that provides us with the tools to practice 21 st century medicine in community settings. Or we can sit on our hands and hope that our restrictive practices and current market dominance will keep out the competition for another 100 years.

\section{Paul Hodgkin}

\section{REFERENCES}

1. Mathers NJ, Hodgkin P. The Gatekeeper and the Wizard - a fairytale. BMJ 1989; 298:172-174.

2. Hagel J III, Brown Seely J. The only sustainable edge: why business strategy depends on productive friction and dynamic specialization. Cambridge, MA: Harvard Business School, 2005. 